\title{
Lutzomyia antunesi, Probable Vector de Leishmaniasis Cutánea en el Área Rural de Villavicencio
}

\section{Lutzomyia antunesi as suspected vector of cutaneous leishmaniasis in the Orinoquian region of Colombia}

Adolfo Vásquez-Trujilloํㄹ Erika Santamaría-Herreño², Angélica E. González-Reina ${ }^{1}$, Luz S. Buitrago-Álvarez ${ }^{3}$, Agustín Góngora-Orjuela ${ }^{1}$ y

Olga L. Cabrera-Quintero ${ }^{2}$

1. Facultad de Ciencias Agropecuarias y Recursos Naturales. Universidad de los Llanos. Villavicencio, Colombia. adolfomvz@yahoo.es, angelicamvz@yahoo.es, agongora60@hotmail.com

2. Grupo de Entomología. Instituto Nacional de Salud. Bogotá D.C. Colombia. esantamaria@ins.gov.co, ocabrera@ins.gov.co

3. Unidad de Entomología, Secretaria de Salud del Meta. Villavicencio. Colombia. lsba960@yahoo.es

Recibido 10 Agosto 2007/Enviado para Modificación 20 Marzo 2008/Aceptado 2 Septiembre 2008

\section{RESUMEN}

Objetivo Definir los posibles vectores de leishmaniasis, teniendo en cuenta su abundancia e infección natural con Leishmania spp. en un área endémica de la zona rural de Villavicencio, Meta.

Materiales y Métodos En la vereda La Reforma, donde se presentaron casos de leishmaniasis cutánea en caninos y humanos, se recolectaron con trampas CDC los flebótomos en el intra, peri y extradomicilio de 15 viviendas. Grupos de 1 a 3 hembras de la misma especie fueron procesados por PCR para la detección de parásitos de Leishmania spp.

Resultados Se capturaron 1304 flebótomos distribuidos en 9 especies de las cuales L. antunesi $(75,6 \%)$ y L. walkeri $(19,2 \%)$ fueron las más abundantes. Otras especies reconocidas como antropofílicas (L. panamensis y L. gomezi), se presentaron con abundancias muy bajas $(<2,4)$. La PCR detectó infección con Leishmania spp. en dos grupos de $L$. antunesi (total= 123 hembras procesadas).

Conclusiones $L$. antunesi por su abundancia e infección natural con Leishmania spp. podría estar involucrada en la transmisión de la leishmaniasis cutánea en un área rural al suroeste del municipio de Villavicencio.

Palabras Clave: Leishmaniasis, PCR, Colombia (fuente: DeCS, BIREME).

\section{ABSTRACT}

Objective Identifying probable cutaneous leishmaniasis vectors in a rural area a few kilometres from the city of Villavicencio, taking the relative abundance of sand-flies and their natural infection with Leishmania spphaving into account.

Materials and Methods A CDC trap was used for sampling sand-flies in and around 
15 dwellings. Pools of three females from the most abundant Lutzomyia species were used for identifying Leishmania spp. by PCR, with OL1 and OL2 primers.

Results 1304 sand-flies from nine species were captured, of which $L$. antunesi $(75,6 \%)$ and $L$. walkeri $(19,2 \%)$ were the most abundant. These was a low abundance of $L$. panamensis and $L$. gomezi anthropophilic species $(<2,4 \%)$. PCR detected Leishmania spp. infection in two $L$. antunesi groups (total $=123$ processed females).

Conclusions Due to the fact that $L$. antunesi was the most abundant species and was found to have Leishmania infection, it may be considered to be the main suspected cutaneous leishmaniasis vector in the rural area being studied. It is recommended that detailed studies of this species' biology (including biting and resting behaviour) should be carried out, aimed at furthering vector control measures.

Key Words: Leishmaniasis, PCR, Colombia (source: MeSH, NLM).

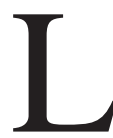

a Orinoquía y Amazonía colombiana comprenden extensas regiones selváticas y de sabana constituyendo alrededor del $60 \%$ de la cobertura total del territorio $(1,2)$. A pesar de las frecuentes alteraciones de los ecosistemas naturales generados por el aumento de migraciones y colonización humana, esta región aún conserva las condiciones que favorecen la presencia y variedad de especies de artrópodos dentro de los cuales revisten importancia en salud pública los vectores de la leishmaniasis. La mayor diversidad de especies de flebótomos típicas de la selva se sigue registrando en esta área, que reúne un 64 \% del total de las especies registradas para el país.

En el departamento del Meta se llevó a cabo la primera notificación de especies de flebótomos para Colombia (3). A la fecha se han reportado para este departamento 35 especies de Lutzomyia de las cuales seis tienen antecedentes vectoriales: L. davisi, L. hirsuta hirsuta, L. yuilli, L. antunesi, L. gomezi y L. panamensis (3-6). Sin embargo, estudios de la ecología o incriminación de los vectores en cada foco específico son escasos (7-8) lo que dificulta la recomendación de medidas de prevención y control.

La forma de leishmaniasis predominante en el Meta es la cutánea y se presenta principalmente en áreas selváticas con conflicto armado. De acuerdo con el Sivigila, en los últimos 4 años (2004-2007) se notificaron 654 casos de leishmaniasis cutánea (LC) únicamente en población civil, siendo los municipios de Macarena, Uribe y Vistahermosa, los que más reportaron casos. El municipio de Villavicencio constantemente notifica casos, la mayoría procedentes de otros municipios; sin embargo, en los últimos años se han reportado casos autóctonos de la enfermedad, a pocos kilómetros del área urbana de Villavicencio. 
Entre julio y noviembre del 2005, se notificaron 13 casos de LC procedentes del área rural de Villavicencio, específicamente de las veredas Apiay, Barcelona, Cocuy y La Reforma, en esta última vereda se realizó búsqueda activa de casos en el mismo periodo de tiempo, encontrando seis caninos y dos de sus propietarios con diagnóstico confirmado de la enfermedad (9). Este brote de LC en personas y caninos, motivó la realización inmediata de un estudio entomológico en el área con el fin de conocer las especies de flebótomos que podrían estar involucradas en la transmisión de la enfermedad, tomando como criterios la abundancia relativa de las especies y su infección natural con parásitos de Leishmania spp.

\section{MATERIALES Y MÉTODOS}

El estudio se realizó en el área rural del municipio de Villavicencio, Meta, específicamente en la vereda La Reforma, la cual está ubicada al suroeste del municipio y separada del casco urbano por aproximadamente $12 \mathrm{Km}$. El terreno es plano, con una altura promedio de $380 \mathrm{msnm}$, destinado principalmente al cultivo de pastos para ganado; también se observan morichales y un bosque de galería que rodea un curso angosto de agua. En la vereda se encuentran dispersas 27 viviendas.

Los muestreos entomológicos fueron realizados al final de la temporada lluviosa y principio de la estación seca, entre noviembre y diciembre de 2005. En 15 viviendas de la vereda, se instalaron trampas de luz tipo CDC, en el intradomicilio y peridomicilio, el primero corresponde a uno de los dormitorios ocupados de la vivienda y el segundo a las áreas abiertas de las viviendas, donde las personas realizan sus actividades cotidianas como solares, cobertizos de animales y patio, hasta $30 \mathrm{~m}$ de la vivienda. Del mismo modo, se instalaron trampas en 12 puntos de un bosque de galería (extradomicilio) a una distancia entre puntos mayor que $200 \mathrm{~m}$. Las trampas fueron ubicadas a 1,5 m de altura con respecto al suelo y activadas de las 18:00 a las 22:00 h, tiempo en el que se había registrado preliminarmente alta actividad de los flebótomos (datos no publicados, Unidad de Entomología. Secretaría de Salud de Meta).

En el laboratorio, los flebótomos machos fueron aclarados empleando $\mathrm{KOH}$ al 10 \% y solución saturada de fenol (10). Las hembras se asociaron a los machos inicialmente identificados de acuerdo a las siguientes características externas: tamaño del quinto palpómero, pigmentación de mesonoto y coxas, longitud de las venas alpha y beta en las alas y longitud del primer flagelómero. La confirmación de la identificación por observación de las espermatecas se realizó en un 
$10 \%$ de las hembras de cada especie, a las cuales se les disecaron y aclararon en fenol los últimos segmentos del abdomen. Las hembras fueron separadas por especie y estado fisiológico en grupos de hasta tres hembras (11) y almacenadas en viales con alcohol al $70 \%$ a $4^{\circ} \mathrm{C}$ (12).

Para la obtención del ADN a partir de los grupos de hembras, se utilizó la resina de intercambio iónico Chelex $100^{\circledR}$ (Bio Rad), siguiendo el procedimiento reportado por Cabrera 2002 (12). La amplificación se realizó utilizando como iniciadores un par de oligonucleótidos universales para Leishmania diseñados sobre un área de la región conservada de los minicírculos de ${ }_{\mathrm{k}} \mathrm{ADN}$ : OL1: GGG GAG GGG CGT TCT GCG AA y OL2: CCG CCC CTA TTT TAC ACC AAC CCC, que producen una banda de $\approx 120 \mathrm{pb}$ (12-15). Los productos de amplificación fueron analizados mediante electroforesis horizontal en geles de agarosa (NuSieve: SeaKem 3:1) al $2 \%$ y visualizados en un transiluminador de luz ultravioleta. Como control negativo se utilizó ADN extraído de tres hembras de L. longipalpis de una colonia experimental. Como control positivo, se utilizó un cultivo de parásitos de Leishmania (Viannia) panamensis (MHOM/CO/87/CL-412). Adicionalmente, se incluyeron como controles positivos muestras de las lesiones de dos caninos y de una persona con LC, ambos de la vereda La Reforma. Como control de especificidad se utilizó un cultivo de Trypanosoma cruzi (MHOM/92/Fch). Los cultivos de parásitos y de flebótomos procedían de los Grupos de Parasitología y Entomología, INS.

\section{RESULTADOS}

Se recolectaron en total 1304 insectos del género Lutzomyia (865 machos y 439 hembras) distribuidos en 9 especies (Tabla 1). L. antunesi fue la especie más abundante (75,6 \%) seguida por L. walkeri (19,2 \%). Las demás especies se presentaron con abundancias menores al 2,4\%.

Tabla 1. Composición y abundancia relativa de especies de Lutzomyia capturadas con trampa CDC en la vereda La Reforma

\begin{tabular}{|c|c|c|c|c|c|c|c|c|}
\hline \multirow[t]{2}{*}{ Especies } & \multicolumn{2}{|c|}{$\begin{array}{l}\text { Intra } \\
n^{1}=15\end{array}$} & \multicolumn{2}{|c|}{$\begin{array}{c}\text { Peri } \\
n=15\end{array}$} & \multicolumn{2}{|c|}{$\begin{array}{l}\text { Extra } \\
n=12\end{array}$} & \multirow[t]{2}{*}{ Total } & \multirow[t]{2}{*}{ (\%) } \\
\hline & b & 8 & है & 8 & $\dot{s}$ & 8 & & \\
\hline L. antunesi & 80 & 42 & 452 & 178 & 138 & 96 & 986 & 75,6 \\
\hline L. walkeri & 9 & 7 & 164 & 61 & 6 & 3 & 250 & 19,2 \\
\hline L. panamensis & 0 & 9 & 3 & 6 & 3 & 9 & 30 & 2,3 \\
\hline L. gomezi & 1 & 0 & 6 & 5 & 1 & 4 & 17 & 1,3 \\
\hline L. saulensis & 0 & 2 & 0 & 9 & 0 & 2 & 13 & 1,0 \\
\hline L. sordeW & 0 & 0 & 0 & 2 & 0 & 1 & 3 & 0,2 \\
\hline L. vattierae & 0 & 0 & 0 & 0 & 0 & 3 & 3 & 0,2 \\
\hline L. barretoi majuscula* & 0 & 0 & 1 & 0 & 0 & 0 & 1 & 0,1 \\
\hline L. chagasi* & 0 & 0 & 1 & 0 & 0 & 0 & 1 & 0,1 \\
\hline Total & 90 & 60 & 627 & 261 & 148 & 118 & 1304 & \\
\hline
\end{tabular}


Para el análisis de infección natural con Leishmania spp. se procesaron 181 hembras de Lutzomyia distribuidas en 78 grupos. Dos grupos de L. antunesi fueron positivos (Tabla 2, Figura 1) ambos de hembras recolectadas en el peridomicilio.

Tabla 2. Infección natural por Leishmania, detectada por PCR, en grupos de Lutzomyia spp. de la vereda La Reforma

\begin{tabular}{lcc}
\hline Especie & $\begin{array}{c}\text { Total hembras } \\
\text { procesadas }\end{array}$ & $\begin{array}{c}\text { Grupos positivos para } \\
\text { Leishmania spp. }\end{array}$ \\
\hline L. antunesi & 123 & $2 \quad(1,6)^{\star}$ \\
L. walkeri & 26 & 0 \\
L. panamensis & 12 & 0 \\
L. saulensis & 8 & 0 \\
L. gomezi & 7 & 0 \\
L. vattierae & 3 & 0 \\
L. sordelli & 2 & 0 \\
\hline Total & 181 & 2 \\
*Porcentaje de infección (aceptando una hembra infectada por grupo)
\end{tabular}

Figura 1. Detección del ADN de Leishmania spp. con los iniciadores OL1 y OL2 en grupos de hembras de $L$. antunesi del área rural de Villavicencio, Meta.

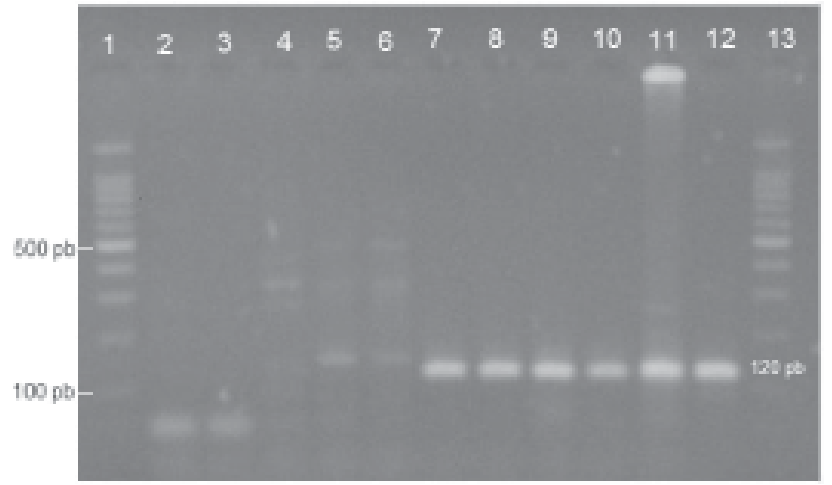

1 y 13: MPM; 2: Control negativo (grupo de tres hembras de L. longipalpis de colonia de laboratorio); 3: Control de reacción (agua Tipo 1); 4: Control de especificidad (cultivo de T. cruzi); 5 y 6: Grupos de L. antunesi negativas para Leishmania spp.; 7 y 8: Grupos de $L$ antunesi positivos para Leishmania spp.; 9 y 10: Muestra de lesiones de caninos con LC de la vereda La Reforma;11: Muestra de una lesión de un paciente con LC de la vereda La Reforma y 12: Control positivo (cultivo de L. (V.) panamensis).

\section{DISCUSIÓN}

Este es el primer reporte de infección natural de L. antunesi con Leishmania spp. en Colombia. En Brasil, esta especie fue encontrada naturalmente infectada con flagelados $(16,17)$ en áreas endémicas para leishmaniasis y es reconocida como una especie antropofílica (18). 
L. antunesi se ha reportado en los 10 departamentos que conforman la región de la Orinoquía y la Amazonía colombiana (4) y en los demás países que integran la cuenca amazónica (18). Aunque es una especie típicamente selvática, en el presente estudio $L$. antunesi se encontró en ambientes asociados al domicilio, especialmente alrededor de las viviendas donde las personas realizan sus actividades cotidianas y donde permanecen los animales domésticos, lo que indica una adaptación progresiva a ambientes modificados por el hombre. Otros reportes recientes indican la presencia de esta especie, aunque con abundancias muy bajas, en bosques cercanos a centros poblados como San José del Guaviare, Arauca, Pto. Inírida y Mato Grosso, Brasil (19-22).

L. walkeri, fue la segunda especie más abundante con una distribución notificada para el centro oriente del territorio nacional, se encuentra generalmente asociada a gallineros (1) por lo que se cree que es más zoofílica y hasta el momento no se ha encontrado infectada con flagelados.

Se registró en $L$. antunesi y L. walkeri un mayor número de machos que de hembras en los tres ambientes muestreados. Esta diferencia puede atribuirse a un mayor fototropismo de los machos de estas especies o a la liberación de feromonas que inducen el comportamiento de agregación y cópula. Un comportamiento similar en la proporción de sexos fue registrada para dos especies pertenecientes a los mismos subgéneros de las especies anteriores, $L$. intermedia (Nyssomyia) y L. migonei (Migonei) en un área endémica para leishmaniasis en Brasil $(23,24)$.

De las otras especies encontradas L. panamensis y L. gomezi son consideradas antropofílicas, probables vectores de LC en Colombia y son frecuentes en áreas periurbanas o rurales muy intervenidas $(25,26)$. Sin embargo su baja abundancia en el área de estudio por el momento no revela un papel importante en la transmisión de la enfermedad, aunque se recomienda realizar un muestreo entomológico más amplio.

En conclusión, por su abundancia en ambientes asociados con el domicilio y su infección natural con parásitos de Leishmania, L. antunesi se constituye en un vector sospechoso de LC en un área rural del municipio de Villavicencio. Con el fin de obtener una mayor comprensión de la epidemiología de la enfermedad y proponer programas efectivos de prevención y control, es prioritario identificar la especie de Leishmania y los reservorios involucrados en el ciclo de transmisión, así como realizar estudios más detallados de la ecología del probable vector incluyendo preferencias alimenticias y comportamiento de picadura y reposo • 
Agradecimientos. A la Secretaria de Salud del Meta, Instituto Nacional de Salud y Universidad de los Llanos, por la financiación del estudio. A las Dras. Martha Stella Ayala y Marleny Montilla, del Grupo de Parasitología (INS), por el suministro de las cepas de referencia de Leishmania y Trypanosoma. A la Dra. Cristina Ferro, por su interés en el estudio. A la comunidad de la vereda La Reforma por su colaboración durante los muestreos.

\section{REFERENCIAS}

1. Toda Colombia. Regiones Naturales [Internet]. Disponible en: http://www.todacolombia.com/ geografia/regionesnaturales.html. Consultado: Julio 2008.

2. Mendoza Morales A. Colombia: Ordenamiento Territorial [Internet]. Disponible en: http:// www.sogeocol.edu.co/documentos/01col.pdf). Consultado: Julio 2008.

3. Antunes PCA. Informe sobre una investigación realizada en Colombia. Rev Fac Med Bogotá Colombia. 1937;6:3-29.

4. Bejarano EE. Lista actualizada de los Psicódidos (Diptera: Psychodidae) de Colombia. Folia Entomol Mex. 2006;45:47-56

5. Vasquez A, González AE, Santamaría E, Góngora A, Cabrera OL, Buitrago LS. Detection of Leishmania spp. in canines and species of the genus Lutzomyia in a rural area of Villavicencio, Colombia. 73rd AMCA. Annual Meeting of the American Mosquito Control Association. 2007; 23(4):449.

6. Montoya-Lerma J, Ferro C. Flebótomos (Diptera: Psychodidae) de Colombia. pp. 211-245. En: G. Amat, M. G. Andrade y F. Fernández (Eds.). Insectos de Colombia. Volumen II. Colección Jorge Álvarez Lleras, No. 13. Academia Colombiana de Ciencias Exactas, Físicas y Naturales; 1998.

7. Barreto M, Burbano ME, Barreto P. Lutzomyia sand flies (Diptera: Psychodidae) from middle and lower Putumayo department, Colombia, with new records to the country. Mem Inst Oswaldo Cruz. 2000;95:633-9.

8. Ferro C, Morales A. Flebótomos de Colombia: Estudios realizados por el laboratorio de Entomología 1965-1997. En: Toro G, Hernández CA, Raad J, (Eds). Instituto Nacional de Salud 1917-1997 Una historia, un compromiso;1998. p. 219-33.

9. Vasquez-Trujillo A. Registro de leishmaniasis cutánea en caninos presentes en zonas periurbana de Villavicencio, Meta y su importancia en la salud pública. Rev Orinoquia. 2006;10 (2):79-84.

10. Maroli M, Feliciangeli D, Arias J. Métodos de captura, conservación y montaje de los flebótomos (Diptera: Psychodidae). OPS; 1997. p.1-67.

11. Santamaría E, Ponce N, Puerta C, Ferro C. Validación de la PCR en la detección de parásitos de Leishmania (Viannia) spp. en Lutzomyia (Diptera: Psychodidae) como herramienta en la definición de especies vectores. Biomédica. 2005;25(2):271-9.

12. Cabrera OL, Munstermann LE, Cárdenas R, Gutiérrez R, Ferro C. Definición de las condiciones de temperatura y almacenamiento adecuadas en la detección de ADN de Leishmania por PCR en flebotominos. Biomédica. 2002;22(3):296-302.

13. Belli A, Rodriguez B, Aviles H, Harris E. Simplified polymerase chain reaction detection of new world Leishmania in clinical specimens of cutaneous leishmaniasis. Am J Trop Med Hyg. 1998;58(1):102-9.

14. Sandoval M, Gutiérrez R, Cárdenas R, Cabrera OL, Ferro C. Estandarización de la técnica de reacción en cadena de la polimerasa para estudios de infección natural de Lutzomyia (Diptera: Psychodidae). Memorias VI Encuentro Científico Instituto Nacional de Salud, 1999 Bogotá. Biomédica 19 Suplemento 1:122. 
15. Pirmez C, Trajano VS, Paes-Oliveira M, Cruz AM, Gonçalves SC, Catanho M, et al. Use of $\mathrm{PCR}$ in diagnosis of human american tegumentary leishmaniasis in Rio de Janeiro, Brazil. J Clin Microbiol. 1999;37(6):1819-23.

16. Ryan L, Silveira FT, Lainson R, Shaw JJ. Leishmanial infections in Lutzomyia longipalpis and Lu. antunesi (Diptera: Psychodidae) on the island of Marajó, Pará State, Brazil. Trans Royal Soc Trop Med Hyg. 1984; 78(4):547-8.

17. Silveira FT, Souza AA, Lainson R, Shaw JJ, Braga RR, Ishikawa EE. Cutaneous leishmaniasis in the Amazon region: natural infection of the sandfly Lutzomyia ubiquitalis (Psychodidae: Phlebotominae) by Leishmania (Viannia) Lainsoni in Pará State, Brazil. Mem Inst Oswaldo Cruz. 1991;86(1):127-30.

18. Young DG, Duncan M. Guide to the identification and geographic distribution of Lutzomyia sand flies in Mexico, the west indies, Central and South America (Diptera: Psychodidae). Mem Am Entomol Inst. 1994;54:1-881.

19. Turriago B, Ferro C, Rodríguez $\mathrm{H}$, Castro F, Cuervo L. Primer registro de Lutzomyia (Diptera: Psychodidae) en el departamento de Arauca. Memorias XXXIV Congreso de la Sociedad Colombiana de Entomología, Cartagena de Indias; 2007. p. 120-1.

20. Flórez L, Ferro C. Especies de Lutzomyia (Diptera: Psychodidae) encontradas en el municipio de Inírida, departamento de Guainía, Colombia. Memorias XIII Congreso Colombiano de Parasitología y Medicina Tropical, Ibagué, 2007. Biomédica 27 Suplemento 2:222.

21. Bejarano EE, Castro M, Perez-Doria A, Hernández-Oviedo E, Vélez A, Vélez ID. Primer Informe de Lutzomyia Franca en el Departamento de Guainía, Amazonia Colombiana, y de Brumptomyia mesai Sherlock (Diptera: Psychodidae) en el Litoral Caribe Colombiano. Neotropical Entomology. 2007;36(6):990-3.

22. Gutierrez de Oliveira A, Bianchi Galati EA, De Oliveira O, Rezende de Oliveira G, Espindola Cabello IA, Cavalheiros Dorval ME, Pecanha Brazil R. Abundance of Lutzomyia longipalpis (Diptera: Psychodidae: Phlebotominae) and urban transmission of visceral leishmaniasis in Campo Grande, state of Mato Grosso do Sul, Brazil. Mem Inst Oswaldo Cruz. 2006;101(8):869-74.

23. Aguiar GM, Medeiros WM, De Marco TS, Santos SC, Gambardella S. Ecologia dos flebotomíneos da Serra do Mar, Itaguaí, Estado do Rio de Janeiro, Brasil. Cadernos de Saúde Pública. 1996;12:195-206.

24. Dílvia FS, Simão DV. Flebotomíneo em fragmentos de Mata Atlântica na Região Metropolitana do Recife, PE. Revista da Sociedade Brasileira de Medicina Tropical. 2005;38(3):264-6.

25. Santamaría E, Ponce N, Zipa Y, Ferro C. Presencia en el peridomicilio de vectores infectados con Leishmania (Viannia) panamensis en dos focos endémicos en el occidente de Boyacá, piedemonte del valle del Magdalena medio, Colombia. Biomédica. 2006. 26 (Supl.1):82-94.

26. Vélez ID, Wolf M, Valderrama R, Escobar DP, Osorio L. Community and environmental risk factors associated wiht cutaneous leishmaniosis in Montebello, Antioquia, Colombia. En: leishmaniosis control strategies: A critical evaluation of IDRC-suported research. IDRC Publications; 1991. p. 261-74. 